\author{
Military Technical College \\ Kobry Elkobbah, \\ Cairo, Egypt
}

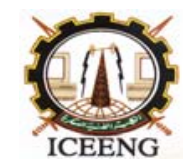

\title{
Analysis Study of Path Rerouting \\ And Handover Schemes for WATM/LEO Satellite Networks \\ A.F.HASSAN, A.ALMOGAZY and H.HEFNAWY
}

\section{ABSTRACT}

\begin{abstract}
One of the major design issues in Wireless ATM and LEO satellite networks is the support of rerouting and handover schemes. The management of mobile terminal handover and rerouting algorithms are the most technical challenges in the recent integrated wireless /LEO satellite networks.

Connection rerouting schemes must exhibit low handoff latency, maintain efficient routes and limit disruption continuous media traffic while minimizing reroute update process to the network switches.

Fast and seamless handoffs are the two factors in the planning to enhance the Performance Evaluation of the QOS for different mobile services.

The objective of this paper is to analyze the different algorithms of connection rerouting and handoff schemes used in wireless ATM networks and LEO satellite networks.

The analysis of this case study creates a new vision for design implementation aspects, which improve the delay and QOS performance of the rerouting and handoff methods used in integrated WATM/LEO satellite networks.
\end{abstract}

\section{Introduction}

Asynchronous Transfer Mode( ATM) is a technology that was defined originally by CCITT (now ITU-T) and ANSI in the US. Their purpose was to define a communication method (or mode) for transporting (or transferring)all forms of information including voice, video and data .Most communications techniques used before ATM's introduction utilized a fixed timing reference .ATM does not, hence the term "Asynchronous".

ATM also provides a connection oriented switched technology that uses dedicated connection to efficiently handle high speed data streams from voice and video applications as well as bursty data from terminal devices. Also ATM effectively supports the high bandwidth requirements of network backbones better than other network technologies.

ATM uses standard size 53 byte cells to transmit digital information at a range of speeds up to $622 \mathrm{Mbps}$. The interleaving of standard size 53 byte cells during statistical multiplexing accommodates bandwidth resources more effectively than conventional packet networks that use frames of varying size. Major advantages of ATM include:

(1) Quality of service (QOS) -based multimedia services for mobile users.

(2)Seamless connectivity with wire line ATM networks/wireless networks.

Over the last few years, the demand for wireless and broadband services has been growing rapidly; Wireless Asynchronous Transfer Mode (WATM)has been a candidate solution for providing broadband wireless services[1].

Recent studies of "WATM" have focused on the communication aspects with ATM as the backbone transport network. Wireless ATM is considered as a wireless access network to interconnect the mobile users to the ATM network. 
Problems in wireless ATM concentrate on the effect of the Packet mode information transport in the wireless environment, which is characterized by unreliable sharing access with finite resource and mobility. Several wireless network architecture have been proposed to interconnect

inherent cellular/Personal Communication systems (PCS), wireless LANs or satellite to the ATM network[1]. The flexibility of the radio interface design is considered to be compatible with existing cellular /PCS systems, and to provide multimedia services such as data, video or integrated services.

Other proposed wireless ATM network architectures focus on the wireless extension of the BISDN terminals for seamless ATM connection.The air interface Design applies ATM cell transport through radio link for broadband integrated services.

The channel coding in the wireless physical layer such as (Forward error correcting (FEC), convolutional coding, interleaving, multi-carrier modulation and diversity reception) are considered to meet the (BER) of individual service requirement.

The design of the Recent Wireless Intelligent ATM(WIATM) network combines three reference models of inherent network: (1) The cellular/Personal Communication System network (wireless),(2) The Air Interface Network (3) The ATM network The evolution from ISDN to B-ISDN emphasizes issues of high-speed network design for broadband integrated traffic with highly diverse service rates. The ATM network features high-speed information transport and high efficiency of the switching capacity for the transport of broad-band integrated traffic by combining the advantages of traditional circuit-switching and packetswitching networks.

In general a WATM network consists of an ATM radio access network and a "mobile ATM core network". [1].

The cellular nature of mobile telephony stems from the subdivision of the serviced area into cells that are covered by electromagnetic signal emitted by the antennas of fixed base stations (BS's).

The mobility of users implies that it's possible for a mobile terminal (MT) to roam from one cell to another, while information transfer over the network is in progress. In order for the communication to continue, It's necessary that the network be capable of transferring the connection from the old cell (source BS) to the new one (the destination BS), this operation is normally referred to as call "Handover" or "Handoff".

The mobility management is one of the critical design issues that arise in WATM networks [2]. In fact, the mobility management consists of two components: Location management which is a two stage process that enables the network to discover the current attachment point of the mobile user for call delivery. The first stage is location registration which the mobile terminal periodically notifies the network of the new access point, allowing the network to authenticate the user and revise the user's location profile. The second stage is call delivery, here the network is queried for the user location profile and the current position of the mobile host is found.

The second component of the mobility management is the Handoff management which enables the network to maintain the user's connection as the mobile terminal continues to move and change its access point to the network[2].

Three stage process for handoff, first involves initiation, where the user, a network agent, or changing network condition identifies the need for handoff. The second stage is new connection generation, where the network must find new resources for the handoff connection and perform any additional routing operations. The final stage is data flow control, where the delivery of the data from the old connection path to the new connection path is maintained according to agreed-upon service guarantees. 
In general, the mobility management aims to achieve, efficient and expedient packet processing, minimizing signaling load on the network, optimizing the route for each connection, and refining quality of service of the handoff mobility management operations.

In WATM networks, extra traffic load may be added to network links due to user mobility and handoff-rerouting .In [1] the study of the effect of user mobility and handoff path rerouting on the traffic distribution in a mobile network environment has been analyzed and evaluated. The analysis has been modeled by flow model which indicates that there are requirements of a higher network capacity and topology reengineering in order to support the same quality of service (QOS) for a mobile service. The dynamic variation of network traffic that affects on the Quality of service has been analyzed with different rerouting algorithms in [3]. A new QOS-based routing algorithm called Backward Hierarchical Routing Algorithm (BHRA)has been proposed to reduce the complexity and maximize network throughput. In[4],an analytical modeling approach has been conducted to estimate the performance of handover protocols making use of handover buffers at the base stations. Which estimate:

(1)The minimum buffer requirement necessary to satisfy the contracted QOS in WATM networks.

(2)The worst case Maximum Burst Size for the ATM connection.

(3) The maximum handover rate allowed by the system to prevent the cumulative effect of consecutive handovers in the connection delay.

Several approximations have been incorporated and the proposed models allow designers to simultaneously take into account numerous system parameters, including handover buffer size, sustainable and peak cell rates of the ATM connection, terminal offered load and time needed to reestablish the ATM connection.

An efficient Handoff Management scheme for wireless ATM network has been proposed in [5]. In this scheme all cells are connected to their neighboring cells by permanent virtual circuit (PVC) and to Access Switch (AS) by switched virtual circuit (SVC's) which are used only for new calls. Rerouting cells are also connected to the AS by (SVC's). If a mobile roams to an ordinary neighboring cell, its traffic path is simply elongated by a PVC connecting the old and new cells. If a mobile roams to a rerouting cell, its traffic path is rerouted to a PVC between the AS and rerouting cell. The proposed scheme indicated that, by using PVC's for handoff cells, there is a guarantee achieving fast and seamless handoff. At the same time the scheme improves the path efficiency by limiting the maximum number of hops that a path can be extended.

In [6], a Stochastic Control of Path Optimization for inter-switch handoffs in WATM networks has been suggested aiming of minimizing the handoff delay while using the network resource efficiency. There is a proposal to determine when and how often path optimization should be performed.

In case of WATM networks, a satellite system could interact with terrestrial wireless network based on the following reasons:

(1)Absorb the instantaneous traffic overload of the terrestrial wireless networks [2].

(2)By using satellite ATM service can be provided over wide geographical area .

(3)Satellite communication systems have a global reach .

The growing interest in the interconnection of satellite and ATM networks is based on the following reasons[11]:

(1)By using satellite ,ATM services can be provided over a wide geographical area , including remote, rural ,urban and increasable area.

(2) Satellite communication systems have a global reach with very flexible bandwidth in demand capabilities. This matches the main characteristics of ATM networks which provide bandwidth-on demand and multimedia services.

(3)Satellite offer flexibility in terms of network configuration and capacity allocation to different sites which use ATM networks in various geographical areas. 
(4)Satellite provide broadcast and multipoint to multipoint capabilities as well as fast network setup which can be useful in deploying multipoint to multipoint communication of ATM networks.

(5)Alternative channels can be provided for connections for which the bandwidth demands and traffic characteristics are unpredictable which may result in maximum resource utilization.

(6) New users can easily be added to the system by simply installing the ATM stations at customer premises.

As a result, possible network expansions will be simple task.

The Low Earth Orbit satellite system can support both the areas with terrestrial wireless networks and areas that lack any wireless infrastructure.

In the latter application area, The LEO satellite would cover regions where the terrestrial wireless systems are economically infeasible to build due to rough terrain or insufficient user population.

LEO satellite is usually defined for those with the altitudes between 500 and $1500 \mathrm{~km}$ above the earth surface [7]. This low altitude provides small end-to-end delays $(20 \mathrm{~ms})$ and low power requirements for both the satellites and the handheld ground terminals. In addition, the intersatellite links (ISL) make it possible to route a connection through the satellites network without using any terrestrial resources [3].

Due to LEO satellites move in reference to a fixed point on the earth .Due to this mobility, the coverage region of a LEO satellite is not stationary. A global coverage at any time is still possible if a certain number of orbits and satellite are used. Coverage area of a single satellite consists of small-sized cells, which are called as spot beams. Different frequencies are used in different spot beams to achieve frequency reuse in the satellite coverage area.

In the next section we classify the different rerouting schemes of Wireless Asynchronous transfer mode (WATM) networks studied so far in the literature. Then we describe handover schemes which are used in WATM networks in section III. The issues of rerouting algorithms for LEO satellite networks are discussed in section IV. In section V the handover problems in LEO satellite systems are discussed . Finally the conclusion is described in section VI.

\section{II: Rerouting Schemes of WATM Networks}

Wireless Asynchronous transfer mode (WATM) is a promising candidate for broadband integrated service wireless networks [8]. However the design of WATM networks faces many technical challenges. In general, Mobility management for WATM is an important factor in developing future wireless networks.

Mobility management deals with transmitting from ATM cell transport based upon the limited and relatively unreliable resource over the wireless channel [2]. Thus it requires the investigation of important issues such as Latency, message delivery connection rerouting, seamless handover, and QOS.

The ATM forum, through the WATM working group, has focused its efforts on developing basic mechanisms and protocol extensions for location and handoff management that address these issues. The forum has specified that the new procedures must be compatible with the current ATM standards in order to be implemented with relative ease and efficiency.

As a result many of the procedures are also compatible with Personal Communication's, satellite, and to mobile IP concepts. In this section, we outlines selected proposed solution for location management, routing scheme, and handoff algorithms for ATM based wireless network.

The key mobility support function in mobile ATM networks is the path rerouting process that is required when the mobile terminal moves from one access point to another [1]. 
Previous studies conducted through both research and standardization activity focused on protocol design.

\section{A. Routing Protocols in PNNI ATM networks}

In [3], a new QOS- based routing algorithm for Private Network to Network Interface (PNNI), ATM network has been proposed. (BHRA) Backward Hierarchical routing Algorithm for ATM networks was compared with conventional routing schemes. BHRA uses an adaptive and iterative path search approach and takes advantage of the PNNI structure. Path selection in network routing has shortest path problems. There is also a problem of routing in a dynamic environment due to the fluctuations in traffic load, link failure, and topology changes. Routing in ATM networks shares some common requirements with QOS-based routing in the internet, in terms of:

(1)Support heterogeneous services, resulting in routing problem subjected to different combination of QOS parameter for different class of applications.

(2)A need for adaptive routing in a dynamic environment due to fluctuation in traffic load, link failures, topology changes and so on,

(3)The complexity of the routing algorithm should preferably be comparable to traditional route computation methods.

There are more challenges in ATM routing due to a wide range services:

(1)How exactly does the routing algorithm cooperate with various resource management functions such as, resource reservation, traffic policing and admission control provided by ATM network?

(2)How to select routing matrices? In case of multiple routing matrices the selected matrices should be orthogonal to each other to avoid redundant information.

(3)What about the scalability of the routing algorithm? In response of that the ATM forum proposed PNNI specifications which consists of routing protocol and signaling protocol, some existing QOS-based routing algorithms were intentioned in [5] as follow:

(a) A heuristic algorithm for the multi-constrained routing problem, which involves two or more additive weight functions. In the case of delay -cost routing, the algorithm first maps the costs to bounded integers and then uses an extended Dijkstra's algorithm to find a solution for the new problem. A feasible path of the new problem is shown to be feasible path of the original problem.

(b)For services with delay guarantee, when a broad class of WFQ-like scheduling algorithms are used, the bandwidth, delay, jitter and/or buffer space constraints is solvable in polynomial time only if the relationship between bandwidth, jitter and delay is taken into consideration.

(c) There is an algorithm combines routing and admission control into a single strategy It assigns each link a length which is an exponential function of the current bandwidth utilization on the link. If no sufficiently short path exists , the request will be rejected $>$

(d) Wang-crowcraft algorithm finds a path for any given constraint on bottleneck bandwidth and propagation delay. First, any link with a bandwidth less than the requirement is eliminated, so that any path in the resulting network topology graph satisfies the bandwidth constraint. Then the path with minimum length is computed using dijkestra algorithm to determine whether a feasible path exists.

(e) A new QOS routing algorithm for ATM networks was proposed by Iwata [3], which is complaint with the PNNI protocol. The algorithm tries to find a path iteratively with different sets of path search approaches until the obtained path meets the requested QOS. The procedure is as follow: First, a candidate path 
that satisfy the QOS requirement is founded. The algorithm then prunes all links that cannot guarantee the requested bandwidth from the whole known topology information, by calculating the equivalent bandwidth of the requested call and comparing it with the available bandwidth for each link. From this reduced set, one or more possible paths are chosen. Second, once a path is found, the source node forwards the message along the path, if a connection is established successfully, the routing function terminates.

(f) Backward Hierarchical Routing Algorithm (BHRA) has some design objectives such as (simplicity, efficiency, scalability, Rerouting-Inter class resource sharing). This algorithm performs better than other routing algorithms such as PNNI sample routing algorithm and Iwata algorithms in terms of connection setup time, computational and storage overhead, all blocking probability and network throughput. The simulation results in [3] showed that call setup time is significantly reduced and the computational overhead and call blocking probability are lower compared to other PNNI routing algorithms. Network throughput is also improved by evenly distributing the traffic among several eligible paths.

\section{B. Connection Rerouting Schemes for WATM}

The need to route the ongoing connection to/from mobile users move among base station is one of the most important challenge in ATM -broadband networks with wireless access links. [9]

Connection rerouting schemes must exhibit low handoff latency, maintain efficient routes, and limit disruption to continuous media traffic wile minimizing reroute updates to the network switches. A typical wireless network with base stations and ATM switches is shown in fig 1 .

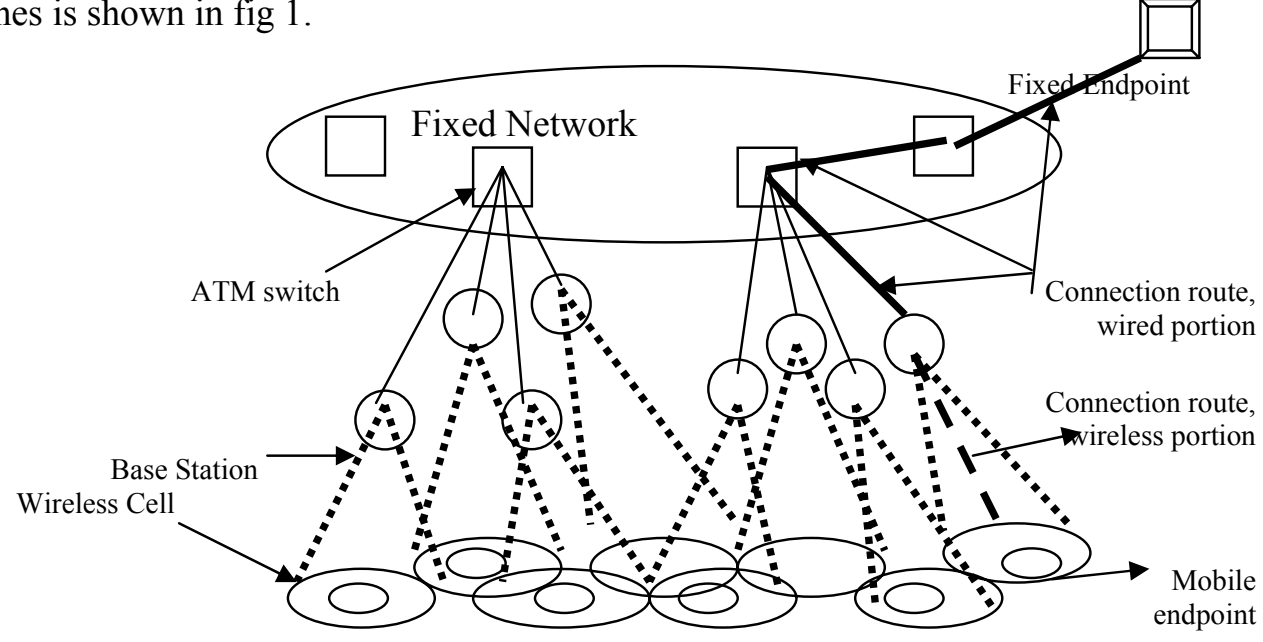

Fig .1. Wireless network with mobile and fixed endpoints

The service area in wireless network is portioned into cells; a cell is the region, which receives its wireless coverage from single base station. In a typical scenario the coverage of the cell s overlaps and the stations are connected to each other and to fixed end points through a wired ATM-based backbone network.

When an end-to-end connection spans both an ATM and a wireless network, it is necessary to dynamically reroute ongoing connections as the mobile user's handoff from one wireless access point to another. Some of the important concerns in performing such connection rerouting are:

(1)Limiting handoff latency. 
(2)Maintaining an efficient route.

(3)Limiting disruption of continuous media traffic.

(4)Limiting network switch update rates due to rerouting.

Limiting handoff latency is essential, particularly in micro-cellular networks where handoffs may occurs frequently and users may suddenly lose contact with the previous wireless access point.

The process of maintaining an efficient route can also potentially lead to disruptions in user traffic that are intolerable for continues media application such as packetized audio and video. Thus, it's important to achieve a suitable tradeoff between the goals of maintaining an efficient route and limiting disruption to continuous media traffic, while at the same time maintaining low handoff latency.

In order to overload the switches, this must be done while keeping the switch updates due to connection rerouting low .Some algorithms using buffers in customized ATM switch that has knowledge of handoff [9]. Five different rerouting schemes has been described and compared. One of the first steps in performing connection rerouting involves the location of "cross over switch".

Each ATM switches an associated channel server which manages the bridging between wired and wireless links and handling handoff requests. There are other servers such as call servers and location servers which perform call processing and location management functions.

In order to reroute a connection, An ATM switch must modify its translation table entries that control its internal cell rerouting. A translation table entry in an ATM switch is a mapping between two 3 tuples, each one consist of a port, (VPI) virtual path identifier, and virtual channel identifier.

The modification of the translation table entry for rerouting can be:

(1) Scheme 1 break-make: dismantle the old entry (break) and then create a new entry. This method results in vulnerability period between these two operations at the switch during which the switch has no translation table entry and drops cells .The algorithm has been able to reduce the length of this period to approximately $3.5 \mathrm{~ms}$.

(2)Scheme 2 make -break: create a new entry (make) and then remove the old entry This results in cells being multicast from the crossover switch to both the new and old base stations for short period of time during the handoff process. Thus there is a possibility that a mobile host will receive a packet from the old base station, switch to the new base station, and receive the same packet as duplicate.

(3)Scheme 3 chaining scheme: Its simplicity extends the connection route from the previous base stations to the new base stations. By provisioning some bandwidth using virtual channel (VC) or virtual path reservation between neighboring base stations, chaining can be accomplished quickly (since the crossover switch will not be involved). However, chaining will typically degrade the end -to -end performance (delay) of connection and connection route is no longer the most efficient.

(4)Scheme 4 chaining with (brake-make): due to the degradation that lead to dropped cells of using chaining scheme only, and using scheme1,2 lead to efficient routing of the connection but involve longer handoff processing delays in order to locate the crossover switch and establish the new route.

(5) Scheme 5 chaining with (make-brake): by doing make-brake after brake-make which perform an immediate handoff using the chaining scheme and if necessary, perform reroute in non real time as in fig 2 . 


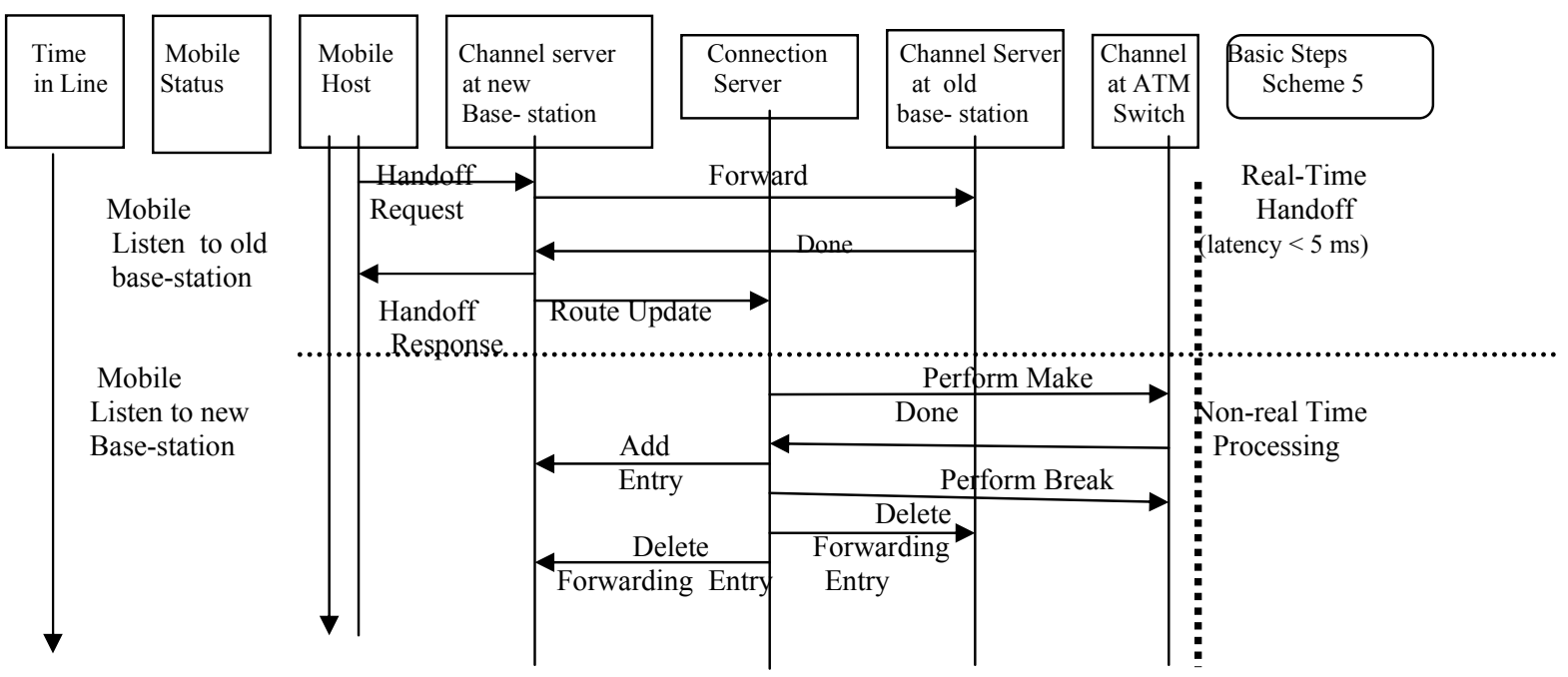

Fig .2. the detailed flow of Scheme 5

As shown in fig 2. the steps involved in scheme 5 as follow:

1- The mobile host sends a handoff request message to the new base station, identifying the old base station and its connection server.

2- The new base station adds local translation table entries for its internal routing.

3- The new base station asks the old base station to forward packets pertaining to the mobile host, instructing the mobile host to transmit / receive through the new base station. The four steps are accomplished in real time (with a latency of $6.5 \mathrm{~ms}$ ) to enable the mobile host to quickly switch over the new wireless link. At that point, the chaining portion of the handoff has been completed.

4- The new base station passes the updated route information to the connection server.

5- The connection server performs necessary QOS computations on the new route.

6- The connection server identifies the best route to the cross over switch, allocates resources along the new route, and sets up a new routing entry in across over switch.

7- Finally, the connection server exchanges messages with the ATM switch, tearing down old routing entry.

The implementation of this scheme was carried out in a testbed consisting of an ATM -based wired network with fixed hosts, ATM switches, and base stations.

The mobile hosts communicate with the base stations using User Datagram Protocol/Internet Protocol (UDP/IP) through a wireless link which replaced by Ethernet. It was noticed that a base station will perform a variety of other functions including call admission control for providing guarantees on forced call termination probabilities, assigning frequency slots or codes, power control, etc.

The definition of handoff latency is the time duration between the following two events at the mobile host (initiation of handoff request and the reception of handoff response). The following table lists the handoff latencies incurred by the five connection rerouting schemes:

Table .1. Handoff Latency in Connection Rerouting Schemes

\begin{tabular}{|c|c|c|}
\hline Rerouting scheme & $\begin{array}{c}\text { Latency for 1 } \\
\text { connections(in ms) }\end{array}$ & $\begin{array}{c}\text { Latency for 2 } \\
\text { connections (in ms) }\end{array}$ \\
\hline Break-make (b-m) & 46.4 & 55.5 \\
Make-break (m-b) & 37.7 & 46.6 \\
\hline
\end{tabular}




\begin{tabular}{|c|c|c|}
\hline Chaining & 6.5 & 7.0 \\
Chaining w/(b-m) & 6.5 & 7.0 \\
Chaining w $(\mathrm{m}-\mathrm{b})$ & 6.5 & 7.0 \\
\hline
\end{tabular}

Out of the five schemes studied, scheme 5, which involved a real time handoff using the chaining scheme and, if necessary, a non real- time reroute using the make-break scheme, seems ideally suited for performing connection rerouting.

The separation of the real time nature of handoffs and efficient route identification in this scheme allows us to perform handoffs quickly, and at the same time, maintain efficient routes in the fixed part of the network By exploiting the centralized route management in earlier proposed connection management architecture, the issues of crossover switch discovery and maintaining the QOS measures for a connection across the handoffs are easily addressed. The proposed algorithm indicated that simple rerouting schemes coupled with connection management architecture are sufficient for supporting continuous media applications over ATM -based wireless networks.

From the experimental test in [9], the chaining with make-brake with addition of simple buffering at the base station is the best performance compared with other schemes, which executes very fast reroutes ( with a measured latency $6.5 \mathrm{~ms}$ )in a real time phase and, if necessary, reroute again in a non real time phase to maintain efficient rerouting , the scheme also results in negligible disruption to both audio ((1-100 chance of a single packet loss at CD-quality audio rates of $128 \mathrm{~kb} / \mathrm{s}$ ) and low bit rate video (2 in 100 chance of a single packet loss for $1-\mathrm{Mb} / \mathrm{s}$ video) traffic during connection rerouting .Therefore, a simple handoff schemes coupled with connection management architecture are sufficient for supporting low -bit-rate continues media application over ATM -based wireless networks.

\section{III-Handover Issues in WATM}

Handoff or (Handover) management enables the network to maintain a user's connection as a mobile terminal continues to move and change its access point to the network.

The three stages process for handoff, first, involves initiation, where the user, a network agent, or changing network conditions identify the need for handoff. The second stage is a new connection generation, where the network must find new resources for the handoff connection and perform any additional routing operations. Under network control handoff (NCHO), or mobile assist handoff (MAHO), the network generates a new connection, finding new resources for the handoff and performs any additional routing operations. The final stage is data flow control, where the delivery of the data from the old connection path to the new connection path is maintained according to agreed -upon service guarantees. [2]. In general the handoff management includes two types: interacell handoff and intercell handoff

The interacell handoff occur when the user moves within the service area (or cell) and experiences signal strength deterioration below certain threshold that results in the transfer of the users calls to new radio channels of appropriate strength at the same base station (BS). Intercell handoff occurs when the user moves into an adjacent cell and all of the terminal's connection must be transferred to a new BS. During handoff process the terminal may connect to multiple BS's simultaneously and use some form of signaling diversity to combine the multiple signals. This is called (soft handoff). On the other hand, if the terminal stays connected to only one base station at a time, clearing the connection with the former BS immediately before or after establishing a connection with the target BS, then the process is referred to a (hard handoff). Handoff concerned issues such as; efficient and expedient packet processing minimizing the signaling load on the network; optimizing the route for each connection, efficient bandwidth reassignment, evaluating existing methods for standardization, and referring quality of service for wireless connection. 
In the following sections we introduce a view on the different handoff schemes used in both WATM and LEO satellite networks, which is moving towards the integrated wireless networks.

\section{A. Seamless Handover Protocol with Buffering at Base Station}

Performance evaluation of seamless handover protocols is an essential step to compare multiple approaches to designing and controlling the WATM system as shown in [4]. In particular, when a user MT moves from one cell to another, all ATM connections originating or terminating at the MT must be rerouted from the source base station (BS) to the distention BS. During this transition the end user may experience the following:

(1)Cell losses and delay variations due to the temporary interruption of the wireless link.

(2) Out of order cell delivery due to rerouting of the connection through the network. Handover protocol must be designed so as to guarantee virtually loss-free and in sequence delivery of ATM cells to end users with minimal delay increases, while ATM connections are being rerouted. A straightforward solution to this problem consists of handshake protocol between the connection and user's is being rerouted. However, if the round trip time of the connection is relatively long, the traffic interruption due to the handshake may not be acceptable for application such as voice and video that have stringent quality of service (QOS) requirements. Moreover, the user at the other end of the connection must be notified of the occurring handover, thus requiring a global upgrade of the protocols at all nodes of the wired network.

Some of the proposed seamless handover protocols require cell buffering at both the destination BS and the neighboring ATM switch. Others require cell buffering only at the destination BS with the aim to minimize the necessary upgrades of the ATM switches in existing networks.

In [4] a comprehensive analytical model to obtain approximate but accurate estimates of the performance of handover protocols and buffering policies at the destination base station (BS). The proposed model relies on both the representation of the distinct phases that characterize the call handover and the consideration of large number of system parameter that includes:

(1)The bursty nature of data and the traffic load generated by the application running at the terminal.

(2)The average random time necessary to reestablish the ATM connection path through the network.

(3)The average random time necessary to establish the new wireless link.

(4) The sustainable cell rate (SCR) and the peak cell rate (PCR) of the ATM connection.

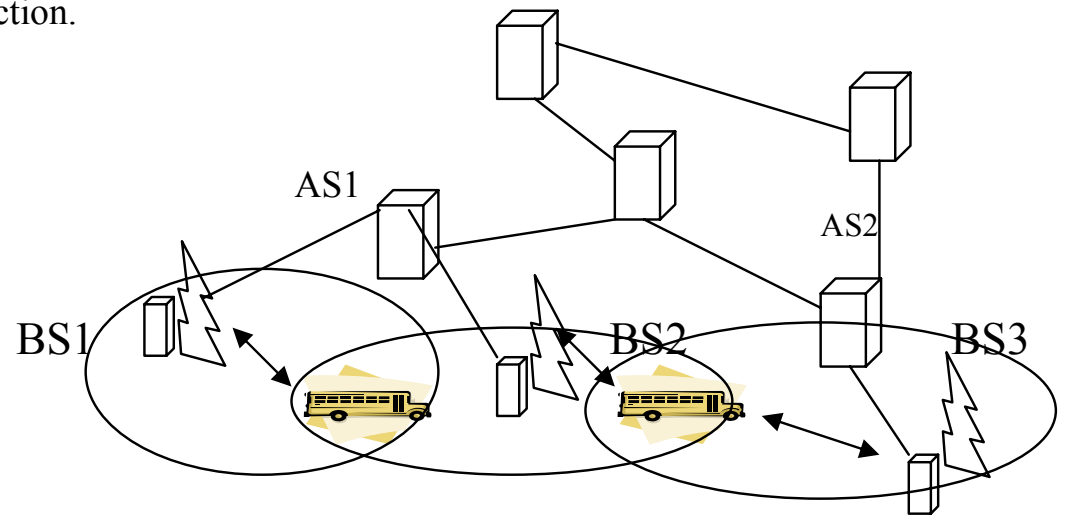

Fig .3. the WATM Architecture [4] 
In fig 3. an example of WATM architecture, three types of nodes characterize the network as follow:

(1) BS's that represent the access points to fixed network.

(2) MT's that freely room in geographical area covered by the cellular network

(a terminal that moves from one BS to another must request a call handover for each of its established ATM connections).

(3) ATM switches, that are part of the wired network. The ATM connection exploiting the non -real time variable bit rate (nrt-VBR), but the proposed approach can be modified to cope with other service classes. Transmission between MT and BS is achieved by means of wireless interface capable of transmitting ATM cells. The advantage of this approach is the resulting transparent connection between the wireless link and the wired ATM network that does not require any ATM adaptation layer (AAL) at the BS. According to this transmission scheme, cells generated by the MT are transmitted over the up stream ATM connection. Similarly, cells transmitted by the terminal at the other end of the connection arrive at the BS via the downstream ATM connection and then forwarded to the MT via the wireless link. Fig (6) shows the case of a MT roam s from BS1-BS2 and from BS2-BS3 incremental path is used to update the path of the connection associated with the MT.

The scope of the seamless handover protocol is to guarantee loss-free and in-sequence cell delivery to the end users during handover. The sequence of events in the seamless handover protocol is as shown in fig 4.

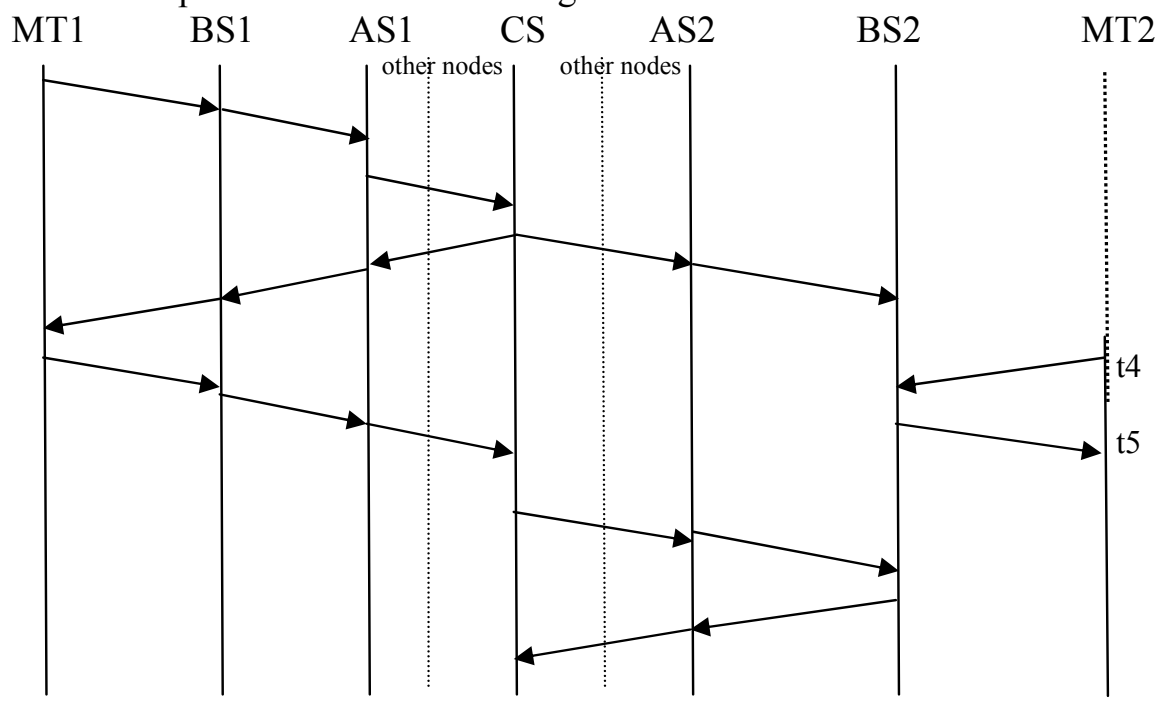

Fig 4. Temporal diagram of the seamless handover protocol MT1 (MT2) indicates that MT is connected to BS1 (BS2) [4]

These events include action taken by the MT, the designated CS, and the destination BS (indicated as BS2). The MT sends a handover request (HOR) to the current BS (BS1) notifying the intention to change BS. While waiting for the network reply, the MT continues to transmit and receive cells using the current connection path. During time interval $\{\mathrm{T} 1, \mathrm{~T} 2\}$, an ATM switch is chosen to be the CS and informed of the handover request. After verifying that the necessary network resources are available at BS2, the CS notifies the MT that the handover confirm (HOC). Soon after, the cross over switch is ready to initiate the traffic rerouting over the incremental path.

Upon reception of the handover confirm, the MT has received all the down stream cells transmitted via BS1. Consequently, it disconnects from BS1 by sending the end upstream data flow (EDF) message and attempts to establish a new wireless link with BS2. Clearly, until the new wireless link is created, cells cannot be transmitted between the MT and the BS's. During this time interval indicated as $\{\mathrm{t} 3, \mathrm{t} 4\}$, it may 
be necessary to store data cells at both MT (upstream traffic) and BS2 (downstream traffic). Downstream cells are buffered if $\delta 1<\delta 2$, i.e. downstream cells arrive at BS2 before the new wireless link is established. Similarly, if $\delta 2<\delta 4$, i.e, the start data flow (SDF) message reaches BS2 earlier than the upstream ready (USR) message, Upstream cells are buffered at BS2 until the new upstream connection is established via the CS. The call arrival rate at the BS buffer is kept below the cell departure rate. However, traffic shaping device used to maintains the terminal transmission rate below the sustainable cell rate (SCR). Cell stored in the BS buffer are transmitted at the connection peak cell rate (PCR). By controlling the gap between the PCR and the SCR, with SCR $<$ PCR it is thus possible to determine how handover buffer will be emptied. Two alternative polices are considered to control the handover buffer:
(1)Dedicated buffer (DB).
(2) Buffer sharing at the base station (B2S2).

In[4], a dedicated modeling analysis has been suggested for both policies with some assumption as:

(1)All delays in the system must be taken to be exponentially distributed random variables, as required by General Stochastic Petri net (GSPN) models.

(2)The time between two handover requests from the same MT is assumed to be longer than the time required to complete the handover cycle.

(3)The propagation delay in the WATM network is negligible with respect to the time necessary to the rerouting of the ATM connection.

(4)The MT average offered load is denoted by lo Mb/s. where the message size can vary from very few cells to ten's cells.

(5)A representation at the message level is used to limit the growth of the number of states in the underlying Markovian process.

The simulation and analytical results has been conducted. The results indicated that the proposed models are practical and flexible framework for estimating the following:

(1)Minimum buffer requirement necessary to satisfy the contracted QOS in WATM networks.

(2)The worst case MBS (Maximum Buffer Storage) for ATM connection.

(3)The maximum handover rate allowed by the system to prevent the cumulative effect of consecutive handovers on the connection delay.

\section{B .Stochastic Algorithm}

Stochastic algorithm to control of the path optimization for inter-switch handoffs in WATM network has been suggested in [6]. With the aim of minimizing the handoff delay while using the network resource efficiently, the two-phase handoff protocol uses path extension for each inter-switch handoff, followed by path optimization were analyzed. The determination when and how often path optimization should be performed is one of the key factors for enhancing the performance of handoffs in wireless ATM networks. Two methods based upon a partial connection reestablishment are the path extension and path rerouting schemes as in fig 5 .

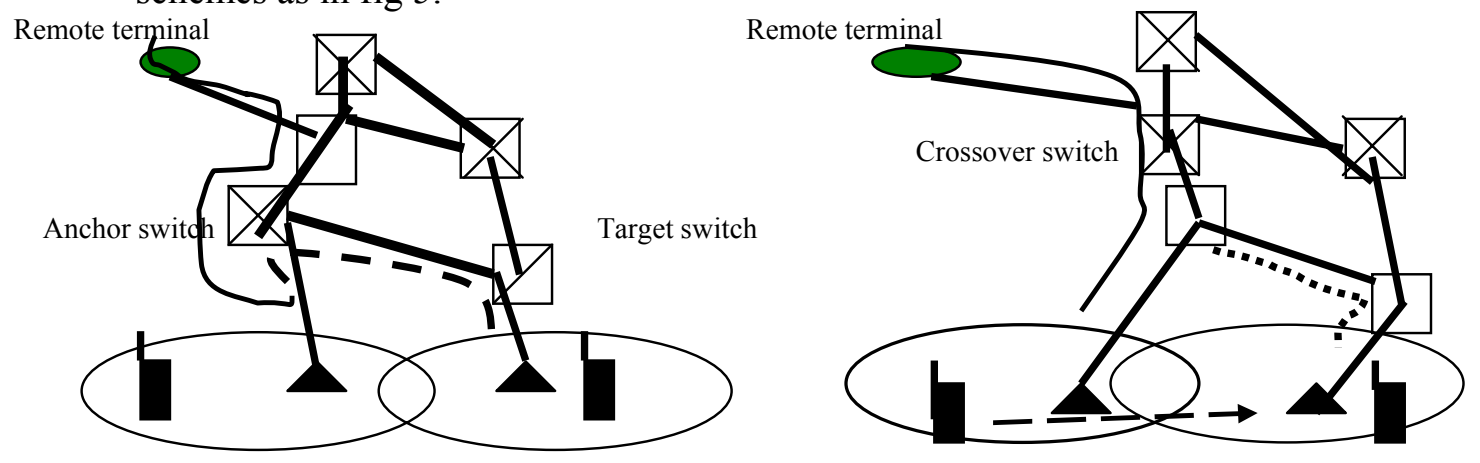




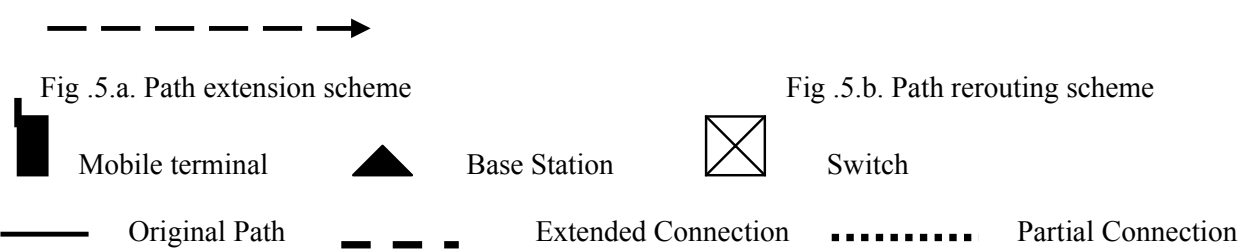

In fig 5.a. the switch to which the original and new base stations are connected are usually referred to as anchor switch and target switch respectively, the path extension method extends the connection from the anchor switch to the target switch during the handoff.

In fig .5.b path rerouting, any switch along the original connection can be selected to setup branch connection to the target switch. The switch chosen to perform this function is usually referred as "crosses over switch".

This path may not be the optimal one, so, in [6] it's a definition of an optimal path as the best path among a set of feasiable paths that can satisfy the prescribed end QOS constraints.

To ensure the seamless path optimization, three important issues need to be address:

(1) How to determine the location of the crosses over switch?

(2) How can the service disruptions be minimized during path optimization?

(3) When and how often should path optimization be performed?

The answers to these question analyzed in details in [6], the analysis was based on when and how should path optimization be performed with the fact that path optimization does not have to be performed after each inter-switch handoff. In addition, if there are large number of mobile -users with high movement patterns, performing path optimization after each path extension will increase the processing load of certain switches and signaling load of the network.

The decision to perform path optimization should be based on several factors, including, the amount of network resources (BW) utilized by the connection, QOS requirements, the remaining time of the connection and the signaling load of the network.

In [6], the path optimization problem is formulated as a semi-Markove decision process. Link cost and signaling cost function are introduced to capture the tradeoff between the network resources utilized by a connection and the signaling and processing load incurred on the network.

The objective is to determine the optimal policy which minimizes the expected total cost per call. A general model has been conducted with the following features:

(1) Different link cost function can be assigned to different service classes.

(2 )Different signaling cost functions can be used based on complexity of the path optimization procedures and the signaling load of the network.

(3)The time between inter-switch handoffs can follow an arbitrary general distribution.

\section{The Model Formulation (Semi- Markov decision process model)}

The model formulation of the path optimization problem is based on a semi-Markove decision process which defined a (decision epoch), that the time instants which network decide after path extension to perform path optimization. The action set $\mathrm{A}=$ $\{\mathrm{NPO}, \mathrm{PO}\}$ represent the action to perform Path Optimization (PO) or Not perform Path Optimization (NPO). The action chosen based on the current state.

The link cost function are taken in consider which reflects the amount of network resources used during the connection life time, while the signaling cost function captures the processing and signaling load incurred on the network due to the path extension and path optimization . 
(The signaling costs are incurred only at the decision epoch, while the link cost is incurred continually over the call lifetime).

The expected total cost per call has been identified in [6] as:

$$
\begin{aligned}
& v^{\pi}(s)=\mathrm{E}_{s}^{\pi}\left\{\sum_{n=o}^{\phi(t)} b\left(X_{n}, y_{n}\right)+\sum_{n=0}^{\phi(T)-}\left[\left(\sigma_{n+1}-\sigma_{n}\right) f\left(X_{n}\right)\right]+\left(T-\sigma_{\phi(T)}\right) f\left(X_{\phi(T)}\right) \ldots\right. \\
& v^{\pi}(s)=\mathrm{E}_{s}^{\pi}\left\{\sum_{n=0}^{\infty} e^{-\mu} \sigma_{n} c\left(X_{n}, Y_{n}\right)\right\} \ldots \ldots \ldots . .(2) \\
& C(s, a)=b(s, a)+E_{s}^{a}\left\{\frac{1}{\mu}\left(1-e^{-\mu \tau}\right) f(s)\right\} \ldots . .(3)
\end{aligned}
$$

The first term in equation (1) represent the total signaling cost, the second term is the link cost at occurring at rate $f\left(x_{n}\right)$ between decision epoch $\mathrm{n}, \mathrm{n}+1$, and the third term correspond the link cost at rate $f\left(x_{\phi}(\tau)\right)$ between decision epoch $\phi(\tau), \tau$.Equation (2) represent the modification of equation (1), while equation (3) represent the expected total cost between two decision epochs at given system state $\mathrm{S}$,

To simplify the analysis, two assumptions are made, first, the distribution of the time between inter switch handoff is independent of the state of action chosen. Second, assume the mobile terminal is communicating with a remote terminal which is stationary, (Mobile- to fixed stationery).

\section{State transition probability function}

Since the end to end delay is proportional to the number of links of the path, a a sub optimal path with a large number of links not only increases the delay but also increases the call blocking dropping probability and the congestion level of the network.

It was proposed that whenever the number of links in a connection is greater than or equal to (L-M) and there is an inter-switch handoff, path extension is performed followed by path optimization with certainty, assume $(\mathrm{K}=\mathrm{L}-\mathrm{M})$, then the current state $(\mathrm{i}, \mathrm{j}, \mathrm{k})$ action set,

$$
\begin{aligned}
\mathrm{A}(\mathrm{i}, \mathrm{j}, \mathrm{k}) & =(\mathrm{NPO}, \mathrm{PO}) & 1 \leq \mathrm{k} \leq \mathrm{K} \\
& =(\mathrm{PO}) & \mathrm{K} \leq \mathrm{k} \leq \mathrm{L}
\end{aligned}
$$

Thus after each path extension, path optimization may be performed if the number of links is less than $\mathrm{K}$, while path optimization is performed with certainty whenever the number of links is greater than or equal to $\mathrm{K}$.

In ATM networks, source routing is being used for all connection setup requests, That is, the source switch selects a path based on topology, loading and reachability information in its database. In large networks it's more appropriate to model the number of links of a path between two end points in a probabilistic manner. On the other hand, for small networks with periodic routing information update, the number of links of a path between the two end- points can be modeled in a deterministic manner.

\section{3 . Cost function}

For each extension events, the network incurs a fixed signaling cost Cpe $>0$ and variable signaling cost hpe $(\mathrm{m})$, where $\mathrm{m}$ represents the number of links increased during path extension. and cpe, hpe capture the cost of setting up the extended path between anchor and target switches.

For each path optimization performed, the network incurs a fixed signaling $\mathrm{Cpo}>0$ and variable signaling cost hpo (L) where $\mathrm{L}$ represents the number links reduced during path optimization, thus, two terms capture the cost of:

(1) Locating the cross over switch.

(2) Setting up the new branch connection. 
(3) Terminating the old branch connection.

(4) Updating the connection server about the status of the existing route.

Then the cost function in case of NPO chosen is:

$C(i, j, k, N P O)=C_{P E}+\sum_{m=1}^{M}\left[h_{P E}(m)+I_{1} f(k+m)\right] p(m / i, j) \ldots \ldots$

The function $C_{P E}+\sum_{m=1}^{M} h_{P E}(m) p(m / i, j)$ is the expected signaling cost for path

extension, given that the location of the anchor and target switches is I and $\mathrm{j}$, respectively.

For path optimization, we assume the number of links of the optimal path is always less than or equal to the number of links of the current path, and less than K, If the action PO is chosen, then the cost function

$$
\begin{aligned}
& C(i, j, k, P O)=C_{P E}+\sum_{m=1}^{M} h_{P E}(m) p(m / i, j)+C_{p o}+ \\
& \left.\sum_{m=1}^{M} \sum_{n=1}^{(k+m) \wedge\left(K^{-1)}\right.} h_{P O}(k+m-n)+I_{1} f(n)\right] p(n / i, D) p(m / i, j)
\end{aligned}
$$

\section{Implementation Aspects}

For each mobile connection, during its connection setup phase, the network controller assign the cost functions based on the service class and the signaling load of the network. Different service classes with different bandwidth requirements are assigned different link cost functions to reflect the network resources consumed.

The assigned signaling cost function reflects the complexity of the path optimization procedures and the current signaling load of the network.

By keeping the mobility profile of each user (movement history and call history), the average time between inter-switch handoff as well as the average duration of the connection can be estimated.

- Given the input parameters (cost functions and various distributions); the value iteration algorithm can be used to determine the optimal policy. The optimal policy is then stored in a tabular format. Each entry of the table specifies the minimum number of links to initiate path optimization for specific pair of anchor and target switches.

-Whenever there is an inter-switch handoff, the network performs a table lookup at the corresponding anchor and target switch entry.

- Path optimization is performed if the number of links is greater than the threshold.

- The optimal policy table needs to be updated when there are changes in the network topology or signaling load in the network.

The numerical results and simulation model as shown in fig 6 . indicated that by using the optimal policy, the total cost is decreased with the number of reduced link increased and gives a lower expected total cost per call than 4 heuristic Algorithms as in [6], and by using optimal policy the mobile connections maintain a good balance the network resources utilized and the signaling load incurred on the network during the life time. Also, the optimal policy is relatively insensitive to the change of the average time between inter-switch handoffs. If there is uncertainty in estimating the average call duration, it may be better to overestimate the value in order to reduce the cost ratio difference. 


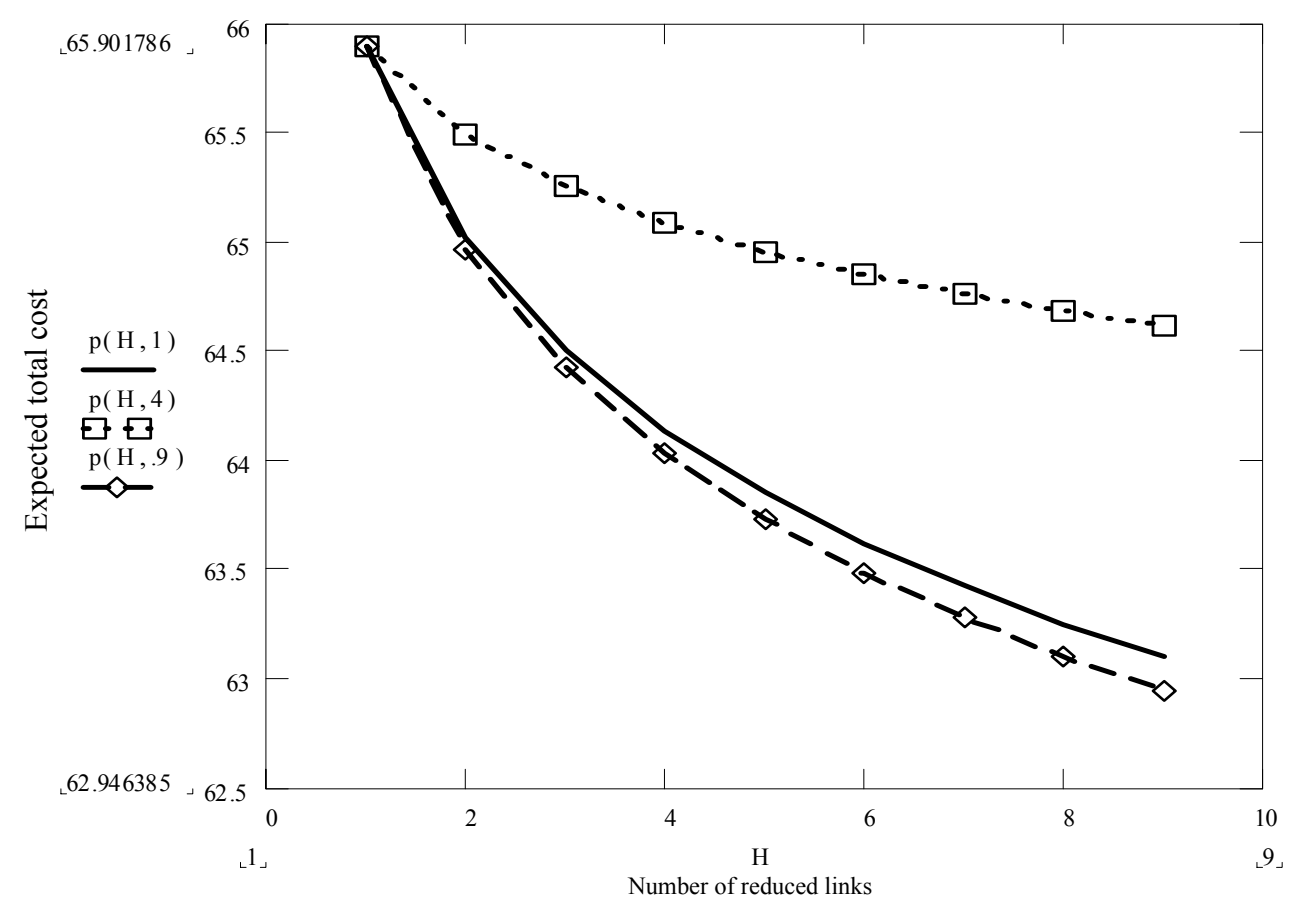

Fig .6. The expected total cost versus number of links in optimal policy

\section{An Efficient Handoff Management Scheme for Mobile WATM}

In [5] a new handoff management scheme for WATM network has been proposed. In this scheme, all cells are connected to their neighboring cells by permanent virtual circuit(PVC's) and to the access switch (AS) by switched virtual circuits (SVC's) which are only for new calls. PVC's also connects some carefully chosen cells, called rerouting cells, to the AS.

PVC's are only used for handoff calls, a system parameter $\gamma$ was defined as the handoff counter in each mobile, each time the mobile makes a handoff, and the counter is increased by one. Each time the traffic path is rerouted, the counter resets to zero. If a mobile rooms to rerouting cell, its traffic path is rerouted to the rerouting cell immediately.

If the mobile rooms to the ordinary cell and the handoff counter is less than the system parameter $\gamma$, the traffic path would be rerouted to the new call via the rerouting cell in the same cluster.

The handoff schemes for WATM networks can be classified into 4 categories:
(1) Full establishment
(2) Connection extension
(3) Incremental reestablishment
(4) Multicast establishment

The new handoff scheme has the advantages of both the connection extension and the incremental reestablishment schemes. On one hand, it enables fast handoff by path elongation using PVC, but at the same time, it improve the path efficiency by limiting the maximum number of hops that a path can be extended. On the other hand, it allows path rerouting to efficiently utilize the network resources. Also, since rerouting is restricted to certain types of handoff calls (handoff counter $=\gamma$ or rerouting cell is hit), the processing load is much less than that in the incremental reestablishment scheme. 


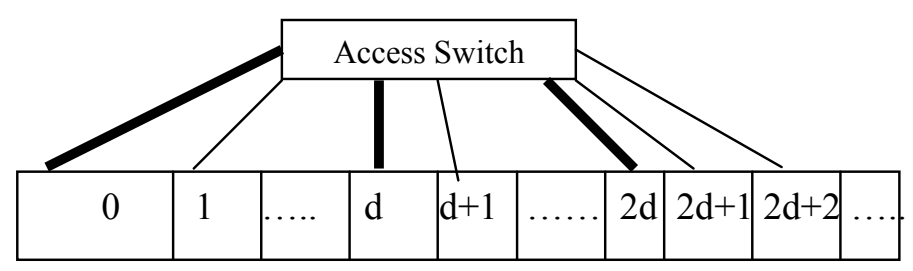

SVC+PVC

- SVC

Fig .7. The architecture of wireless ATM networks in liner environment [5]

\section{Scheme Description}

It was consider the liner environment, as in fig (7), some cells, say cell $0 . d, 2 \mathrm{~d}, 3 \mathrm{~d}, \ldots$ are assigned to be rerouting cells. Between rerouting cells and the AS, only there are SVC's and PVC's. Between those ordinary cells and AS, only SVC's can be established for new calls. Between a pair of neighboring cells, there are PVC's for handoff calls.

If a mobile rooms into an ordinary cell, the traffic path is elongated by a PVC's between the current and the new cell. If a mobile rooms into a rerouting cell, the traffic path would be rerouted directly to the rerouting cell.

Here, it gathering all rerouting of handoff calls to some rerouting cells, thus it obtains some statistical multiplexing gain and reduce the required number of PVC's between cells and AS.

1- It's assumed that a specific connection scenario between AS and 4 base stations with mobile.

2- The important issue for WATM is the presence of buffer at each PVC to preserve the new route.

3- Two-connection path between the mobile user and AS, (UEC) Uplink End Connection and (DEC) Down link End Connection.

4- To control in (CVD) cell delay variation, a scheduling algorithm for jitter compensation should employee at AS. In this system the (LOT) local delay time is assigned for each node during the route establishment process and the (CDV) will improve by using this algorithm.

5- Due to each mobile can keep its path record .So, in case of pass through AS, BS1, BS2, BS3, it keep the path in its buffer. In case of return back to BS2, hence the looping occurred.

\section{Performance Analysis}

Some parameters have been defined as, call holding time, call arrival time and call sojourn time. And the main equations to obtain the handoff call arrival rate equation, the equation of the average number of SVC for each cell, the average number of PVC in case of moving the mobile.

The analysis for liner and planner environment was conducted and the numerical results related to the blocking probability of calls and handover calls indicated that there is an improving in the path efficiency. By suitably selecting the hop limiting parameter $\gamma$ allows most handoff calls to be handled by the involved base stations using PVC's, the added processing node to the network due to the frequent handoffs can therefore be kept low. An analytical model, shown that the scheme required less VC's than VCT and connection extension schemes.

\section{Dynamic priority Queuing of handover calls}

In mobile communications, the number of mobile users that crossing the cell boundaries are increasing, hence the proliferation of handover calls [14]. 
The way that handover calls are handled has direct impact on the QOS provided to the mobile user. Various methods have been devised to prioritize handover calls over new calls. Some approaches proposed certain number of channels is used extensively for handover calls and only queuing of (new) calls is investigated. That minimizes HO blocking probability for $\mathrm{HO}$ calls and increase total carried traffic in the network.

In a modified approach, an analytical frame work of GCM with first in first - Out (FIFO) queuing of $\mathrm{HO}$ calls and NO Queue for new calls. Which reduces the number of dropped handover calls?

The proposed system model in [14] employs a two dimensional (2-D) Markove chain approach and differs from the previous work as:

(1) Two classes of priority of $\mathrm{HO}$ calls are considered.

(2) The transition time required between these priorities is taken into account.

(3) The event that a $\mathrm{HO}$ calls could finish its call while waiting in the queue is captured

by the model.

The dynamic queue of HO calls was not only based on RSS (received Signal Strength), but also on the velocity at which MU is moving, hence the rate of change of RSS.

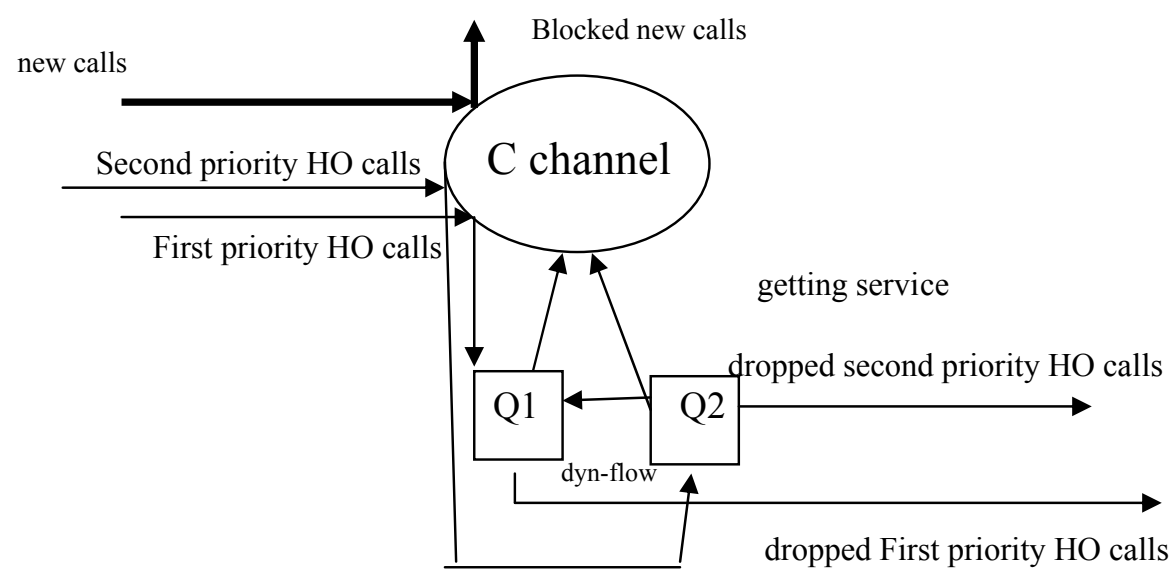

The described model assumes that:

Fig .8. Queuing Model [14]

- $\quad$ There are $\mathrm{C}$ server available (the number of channels per cell)

- $\quad$ The new HO calls get serviced if there is a free server ( free channel)

- If all servers are busy, the new calls are blocked, while HO calls are stored in the queues according to their priorities.

- $\quad$ Consider finite storage for the queue ( $\mathrm{H} 1, \mathrm{H} 2$ for first and second priorities)

- By using $\mathrm{M} / \mathrm{M} / \mathrm{C} / \mathrm{K}$ queuing system, in the case that there are free channels in the cell, new calls or handover are get services.

- When all channels are occupied, new calls are blocked where handover calls are queued in their respective queue according to their priorities. If the queues are full, HO call arrival are blocked.

The analytical framework for dynamic priority for HO calls was presented. The system model indicates that there are 2 scenarios can be analyzed. The first one is integrated voice / data wireless networks, handover voice calls can assigned first priority, and handover data calls are assigned Second priority.

The second scenario could be applied in HO between different network types (vertical Handoff),e.g. (cellular network that use GSM or CDMA and WLAN, It can assign the handover calls of GSM as first priority and WLAN as the second priority.

Some additional parameters can be taken in consider as, queue waiting time, traffic types and the QOS requirements of queued calls. 


\section{IV-Routing Algorithms in LEO satellite networks}

Satellite networks provide a global coverage and support a wide range of services.

Since LEO satellites provide short round-trip delays, they are becoming increasingly important for a real time application such as voice and video traffic.

Satellite networks can be used as a back up for the existing networks. In case of congestion or link failures, traffic can be routed through satellites.

The connection oriented routing has been the focus of the routing protocols in the satellite, some routing algorithms has been proposed earlier to optimize routing paths in LEO satellite network introduced in [10]. The following algorithms presented a package of efficient solutions in LEO satellite routing issues:

(1)The heuristic routing algorithms [11] aims to reduce the number of path handover due to the nobility of satellite.

(2)FSA algorithms use the snapshots of the constellation to optimize the paths.

(3)In satellite over satellite (SOS) networks algorithms present two-layered satellite networks architectures consists of LEO and MEO satellite networks has been proposed.

(4)A QOS -based satellite network routing algorithm were presented that resembles minimum hop routing in Manhattan Street Networks.

(5)The probabilistic routing protocol (PRP) aims to maintain the initial paths as long as possible in order to minimize the signaling overhead.

(6)Due to the effort regarding the Next Generation Internet (NGI) there is an initiative in commercial and also in the military world to use IP routing technology in satellite networks. Darting algorithm delays the exchange of topology update information until it's necessary to send data packet also, this algorithm doe not reduce the protocol overhead.

(7)The datagram routing algorithm aims to route the packets on minimum propagation delay paths.

(8)In [10]a new Multicast routing protocol for datagram traffic in LEO satellite IP networks. In this protocol, non of the existing multicast routing protocols are will suited for LEO satellite networks as :

(a) RPM reserve path multicast.

(b) Distance vector multicast routing protocol (DVMRP).

(c) Multicast routing extension for OSPF (MOSPF).

All the pervious algorithms have some limitation because they employ some form of periodic message exchange to maintain multicast trees, which is not favorable due to the limitation of power on board.

The new scheme is based on datagram routing algorithm. Multicast trees are generated such that the number of branches going out of the satellite is minimized at each step.

The simulation results show that the multicast trees provide delay exceeding the minimum propagation delay by at most $5.5 \%$ on the average. Multicast trees are multiple times shorter than the sum of Uni-cast paths. They also outperform trees generated by Protocol Independent Multicast (PIM), MOSPF, and Core Based Tree (CBT) scheme.

In [12] Different routing strategies for maximizing throughput in LEO satellite networks has been disused. Since, the design of the satellite relies on transmission of burst traffic data by using packet switching technique. Each satellite has limited number of transmitter and limited capacity of storage space for packets.

The problems occur when large number of packet will arrive, a contention is occurring for sending which packet on satellite. So, different routing and scheduling algorithms are used to solve this problem. In particular, it's examined the impact of limited transmitter 
and buffer space on throughput of such networks. The network has been modeled as an $\mathrm{N} x \mathrm{~N}$ mesh network and analyzed throughput performance of the system by:

(1)Study the stability region of the system.

(2)Consider 3 transmitter schemes, Short Hop Win(SHW), Random packet win(RPW)and Oldest Packet Win(OPW) . comparing their throughput performance by using fixed -point analysis for the system Markove chain has been conducted in [12].

-As multiple packets arrive at a particular node in time slot, SHW chooses the one with shortest hop distance to the destination to be transmitted in the next slot.

- RPW where the wining packet is chosen at random and OPW where the wining packet that has traveled the largest distance wins the contention to destination. The analysis proved that when the satellite node has no buffer the analytical and simulation results show that SHW scheme attains the best throughput performance followed by the OPW and RPW scheme.

By using Multicast routing schemes, new packet had lower priority than continuing packets and hence, when contention occurred at node, new packets were prevented from entering the network.

Once packet entered the network .It reached to destination with very high probability. In particular system, the arrival rate of new packets into the network must not exceed the throughput value in order for the system to be stable.

The analysis of ATM-based routing in LEO/MEO satellite networks with intersatellite links has been presented in [13]. Specific emphasis is laid on the design of an ATM -based routing scheme for ISL part of the system. The approach is to prepare a virtual topology by means of virtual path connection(VPC's) connection all pairs of end nodes in the ISL sub-network for a complete period in advance, similar to implementing a set of (time dependant) routing tables. The search for available end-to-end routes within ISL network is based on a modified Dijkestra Shortest Path algorithm (M- DSPA) capable of coping with time variant topology. It could be shown that this strategy is capable of reducing the average number changes for an end to end connection, without significant increase of the path delay. At the same time, one evades significant rearrangement of large traffic streams over time. This approach permits the design of an optimized routing strategy over specific time intervals of the constellation period, and is done once for all, given the constellation geometry and the ISL connectivity.

A major benefit of a low VPC-HO rate is absence of sever delay jitter for large part of typical end -to -end connections which in turns reduces complexity of the ATM cell streams alignment. It has shown that typical maximum end-to-end delay within ISL subnetwork is about $180 \mathrm{~ms}$ for LEONET and $240 \mathrm{~ms}$ for iridium, and the delay jitter is below $20 \mathrm{~ms}$ in more than $90 \%$ of the connections encountering a VPC-HO. Since an end -to-end protocol, will in principle carry out The ATM cell streams alignment the consideration of the interface to the UDL routing task is essential for the global performance evaluation.

\section{Handover Issues in Satellite Systems}

In future generation communication systems, integration among different segments (terrestrial indoor, terrestrial outdoor and satellite) will be necessary if aiming to provide real global and contiguous coverage without interruption.

To achieve integrated or internetworking systems, efficient algorithms to perform Inter-segment Handover (ISHO) have been implemented in [15]. It aims at analyzing ISHO procedure developed in some projects and evaluating their performance for different system configurations utilizing a dynamic constellation simulator in the time domain. 
The mobility management of interworking systems is discussed in details in [2],[15], with the ISHO is a critical issue. Each network can have different access schemes, particular network functionality, and different propagation delay.

The problem is particularly considerable when a satellite system is interacting with the terrestrial one, mainly because of different transmission delays and of synchronization.

The HO procedure is realized in two main phases: Decision phases and execution phase as in fig (9).

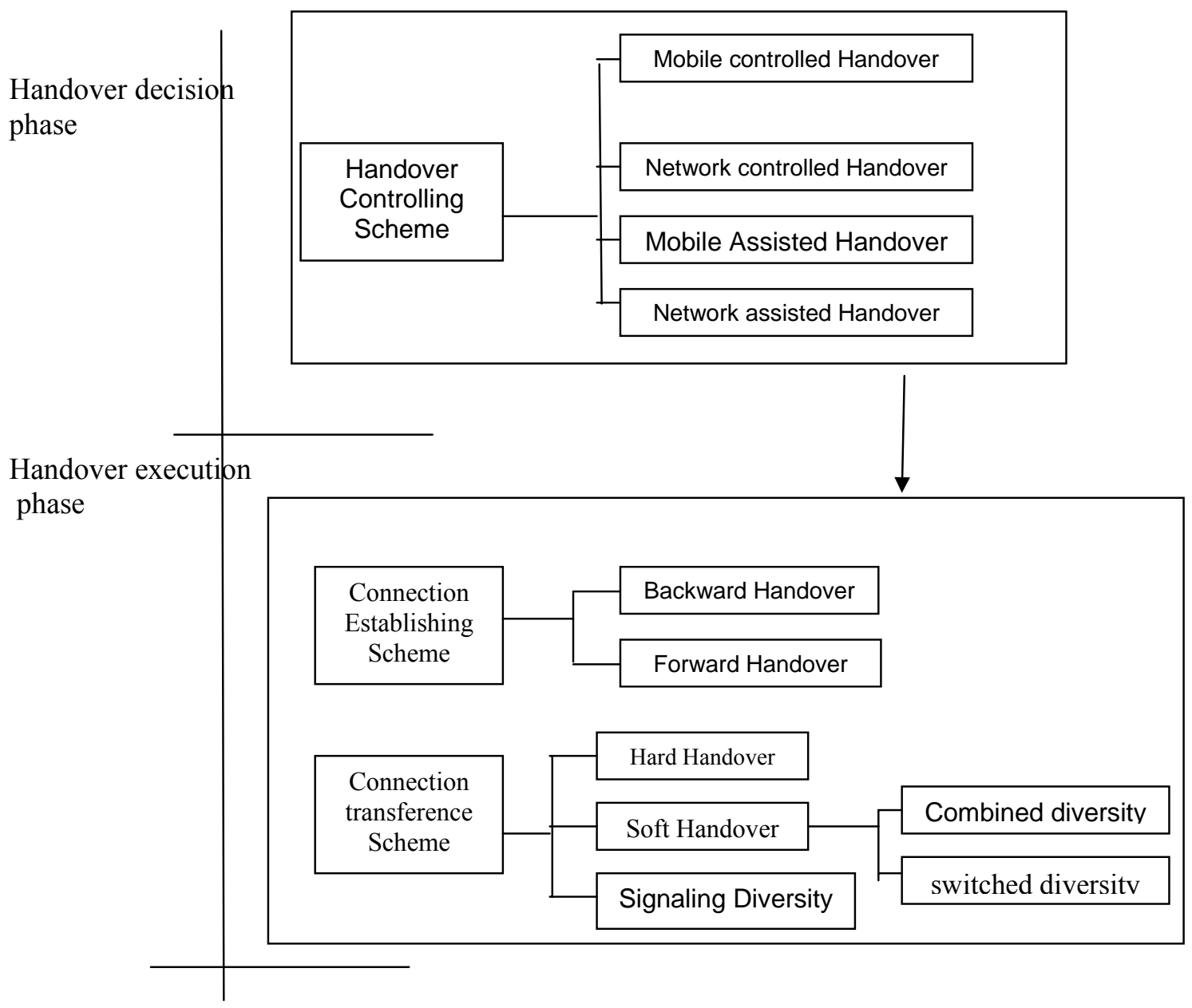

Fig .9. Inter-segment handover strategies

The two phases show different performance in terms of execution delay mainly due to the number of signaling messages. The increase of the relative distance user-satellitegateway enlarges the overall delay difference, especially if the satellite is located in geostationary orbit. The updated handover schemes in LEO satellite network are summarized in the following strategies.

\section{A. ISHO signaling routing strategies}

In [15], the adoptable strategy depends on the combination of controlling schemes and routing strategy. A set of functional entities are evolved in HO. as (measurements functions- Cell management- Decision and initiation function- user profile information - HO criteria function) 
Each combination of controlling scheme and routing strategies depends on which functional entities are available for the system. The analysis in[15] based o the Mobility Management (MM) scheme, several components exchanging messages are involved as ,\{ Mobile Terminal (MT)-Satellite(SAT)- Gateway(GW)- Base transceiver station (BTS)-Core Network(CN).The function of (MT and GW)is to provide connection between Mobile segment (terrestrial or satellite)and fixed networks.

There are 4 signaling scheme to analyze the evolution of signaling routing during a complete handover from earth to satellite and vice verse :

(1) Backward procedure from terrestrial to satellite segment.

(2) Forward procedure from terrestrial to satellite segment.

(3) Backward procedure from satellite to terrestrial segment .

(4) Forward procedure from satellite to terrestrial segment.

The impact of handover occurrence on Packet flow affects, once the HO has been successfully executed the transition from one segment to the other can cause packet misalignment due to the different delay of the two segments that is discussed. In fact the message routed through the satellite segment experience a delay greater than those routed through the terrestrial one due to the path length difference.

A buffer storage can be foreseen to compensate the delay difference and to allow packet alignment. The overlapping can also compensate the temporal gap generated if a $\mathrm{HO}$ in the other direction previously occurred.

Two different scenarios were discussed for the selected procedure (Accord and Rainbow). Performance analysis for ISHO is carried out in 4 communication routes (backward and forwarded procedures from satellite and to terrestrial and vice verse).

An optimized procedure was analyzed aiming to efficient the resource management; it is based on MAHO (Mobile Assisted HO) technique and signaling diversity transference scheme. ISHO from terrestrial to satellite and vice verse was discussed. Also, the simulation scenario in case of GEO, MEO and LEO constellation satellite was conducted and the evaluation parameters (maximum delay and delay range) for each system were analyzed. In general the proposed procedures are better than the classical backward procedure and the forward in the opposite direction. Different HO strategies may be adopted in the two different directions, but it results preferable to adopt the same procedure. Also the delay is in general quite long, especially if high altitude constellations are used, but it can be reduced both optimizing procedures and constellation structure.

\section{B.Queuing policies for handover Requests in LEO satellite Systems}

In [16] ,a mobility model suitable for low earth orbit mobile satellite systems(LEO-MSS's) has been presented, and its statistical parameters have been derived in order to evaluate the impact of the mobility on the performance of the fixed channel allocation (FCA)strategy.

Future global coverage mobile satellite systems (MSS's) will be able to provide the users with communication services anytime and anywhere.

Many strategies have been recently proposed to privilege the handover service at the expenses of new call arrivals in case of terrestrial microcellular systems. The study of the performance of these policies in a satellite contest based on the queuing of handovers, which do not immediately attain service is considered. A 
classical Fixed Channel Allocation (FCA) technique has been assumed: that is each cell has predefined set of resources to satisfy the channel requests in it.

In case of Iridium system there are some preliminary consideration and assumption on LEO-MSS's . Due to the beam forming, the spot beam foot prints are disposed on the earth according to a hexagonal regular layout(side $\mathrm{R}$ ) where $\mathrm{R}=212.5 \mathrm{~km}$. The cellular network irradiated on the earth by the multi-spot beam antenna from a satellite has been considered parallelogram shaped .Due to the high value of the satellite ground track speed $V_{\text {trk }}$ (about $26000 \mathrm{~km} / \mathrm{h}$ in LEO case) the relative satellite user motion can be approximated by only vector $V_{t r k}$; then, mobile stations (MS's) cross the cellular network irradiated by a satellite according to parallel straight lines. Only the voice traffic has been considered, as in classical fixed telephony, new call attempts that do not immediately find available resources are blocked and lost.

The LEO Mobility Model can be summarized as follows;

1- MS's cross the cellular network with the relative velocity, vector $V_{t r k}$.

2- When a handover occurs, the destination cell is the neighboring cell in the direction of the relative satellite user motion.

3- Calls are uniformly generated all over the network.

4- From the call outset in a cell, the related MS travels a distance (depending on $\mathrm{z}$ ) defined as :

a.Uniformly distributed between zero and $h(z)$ if the cell is the source cell of the call.

b.Deterministically equal to $\mathrm{h}(\mathrm{z}) \mathrm{I}$ the cell is a transient cell of the call.

In [16] a derivation of the mobility equations parameters is conducted and the handover process across the seam of the cellular network was analyzed.

Two main approaches of Queuing Disciplines were discussed (FIFO) and the measurement -based prioritization scheme (MBPS). The (MBPS) is measuring the signal received by the satellite. The target is to serve first the request from the MS with a more degraded link. The quality of the link is continuously monitored for each handover request in the queue. The idealized queuing scheme is denoted as (LUI) last useful instant, relies on the fact that when the handover request is queued, the system exactly knows its maximum queuing time $\left(t_{w \max }\right)$. Then the system serves first the most critical handover request .The description of Handover queuing technique with its parameters and discipline was analyzed. The analysis of (FCA-QH) with different queuing strategies is derived by using $\mathrm{M} / \mathrm{M} / \mathrm{S}$ queuing theory. Also the simulation results indicated that the FIFO scheme attains good results very close to the bound given by the LUI technique. Therefore the FIFO solution has to be preferred to the LUI one which would require a greater implementation complexity.

\section{Handover-Independent IP/LEO satellite Mobility management}

The huge need for developing internet protocol (IP) friendly networking technologies that aim to integrate emerging LEO satellite networks with already existing terrestrial IP networks .LEO satellite networks are well characterized by frequent handover occurrences. These handovers largely affect mobility management in LEO satellite networks [17].

The existing IP mobility management protocol (mobile IP), manage the location of the mobile node on the basis of the network topology. In terrestrial mobile networks, only end nodes are subjects to motion while base stations remain fixed. Whereas, in LEO 
satellite networks, both end systems and satellite (base stations) keep on moving .Furthermore, considering the fact that satellite network cover wide areas and should consequently serve a potentially large number of end nods, scalability becomes a major issue.

Conventional IP mobility management protocols require mobile nodes to send binding update requests to the location directory every time a handover occurs.

Given a high mobility of LEO satellite networks, usages a large number of binding update request and consequently affect the scalability of the mobility management schemes.

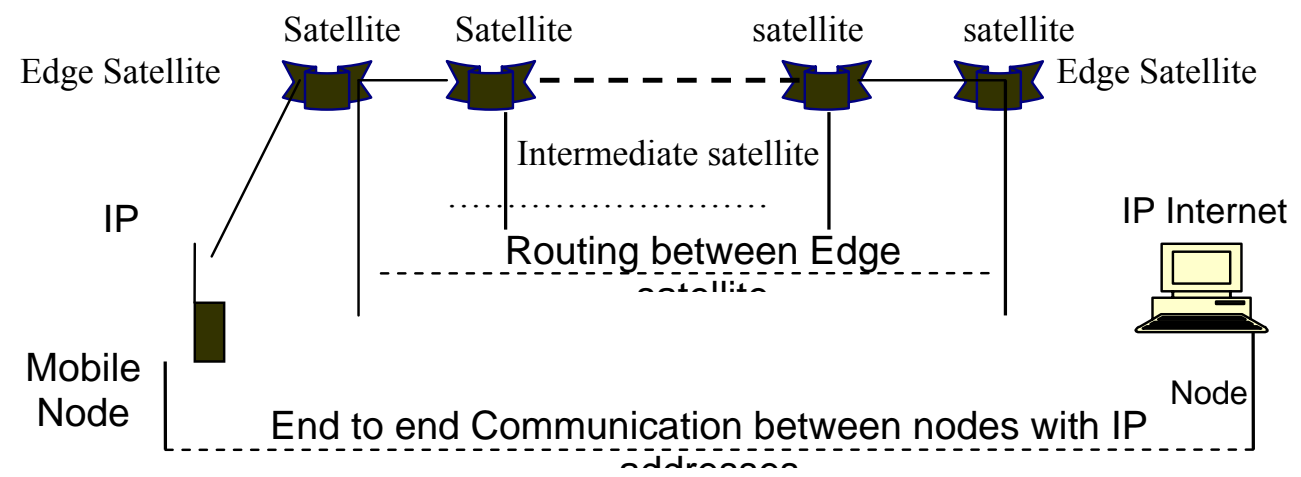

Fig .10. IP/LEO Satellite Network [17].

From Fig (10), an IP communication path consists of three components, namely mobile nodes, edge satellites and intermediate satellites. Each mobile node is assigned a particular IP address .Along a given path, Edge satellite are given a certain number of addresses and play the role of access routers. Intermediate satellites are limited to only transmitting IP packets between edge satellites.

The aim is to integrate IP protocols with LEO satellite networks, while focusing on the interaction between mobile nodes and edge satellites. To make LEO satellites networks entirely independent of terrestrial networks, terrestrial stations are assumed not to be involved in the management of LEO satellite networks.

The analysis of the new schemes of Handover -independent IP mobility management is described in details in [17]. It starts with the advantages of LEO satellite communication and application of IP Networks over LEO satellite. A brief comparison between terrestrial networks and LEO SAT networks from mobility point of view and the requirement of "binding update" between satellite and mobile node should occur frequently. The basic idea behind the scheme is to make IP addresses independent of logical locations and associated to only geographical location information.

The characteristics of LEO satellite networks, the difference from terrestrial networks and the characteristics of Handover occurrence of the moving satellite relative to the ground speed of the satellite while considering the mobile nodes are located in fixed point are discussed and modeled.

The mobility management is the core issues in IP/LEO satellite networks to locate the mobile nodes and to guarantee a seamless data transmission upon change in nodes position It is classified into two main tasks ( binding update) and (data delivery). Considering the possibility of location directory to be geographically too far from mobile nodes, the cost of binding update can become expensive, mainly in a high mobility environment such as satellite networks.

The Handover is a local process that concerns only the mobile node, the old access route and the new access route, whereas a binding update is a global process that may affect other network elements in addition to the three adjacent entities.

The proposed scheme depends on the analysis of the following three issues:

(1)Geographical location mapping to route ID

(2)Cell distribution in satellite coverage. 
(3)Connection setup and maintenance, in terms of the local forwarding scheme and the paging scheme.

The mathematical analysis of mobility management cost which defined the binding update, local forwarding and paging cost is compared with the management cost of mobile IP, P-MIP (paging mobile IP) and the proposed method.

The effectiveness of the proposed method was analyzed by considering the satellite's coverage radius and satellites ground speed are set to $700 \mathrm{~km}$ and $7 \mathrm{~km} / \mathrm{s}$, respectively. Mobile node are assumed to move at velocity $17 \mathrm{~m} / \mathrm{s} \sim=60 \mathrm{~km} / \mathrm{h}$.

The evaluation indicated that to enhance mobility management scalability over LEO satellite networks, a new method was proposed which exploits geographical location information to make the binding update process entirely independent of handovers. In the proposed method, binding updates are issued only when a node crosses the cell boundary and the rate of binding updates is accordingly reduced.

\section{CONCLUSION}

In this paper, a detailed analysis for different algorithms of rerouting and handover issues in both WATM and LEO satellite networks are conducted. It is shown that some rerouting algorithms in WATM networks are used to redesign the network topology by adding more links or increasing capacity in network based on user mobility pattern as First Divergence algorithm. The Stochastic algorithm with optimal policy is used for determining when and how performing path optimization after path extension when the mobile moves to another cell The analysis results indicated that the optimal policy gives a lower expected cost per call which implies that the mobile connection maintains a good balance between network resource utilized and the signaling load incurred on the network during its connection lifetime. The optimal policy is relatively insensitive to the change of the average time between inter-switch handoffs. Four handover algorithms in WATM networks have been discussed, The efficient handoff scheme has the advantages of both the connection extension and the incremental reestablishment schemes. On one hand, it enables fast handoff by path elongation using PVC, but at the same time, it improve the path efficiency by limiting the maximum number of hops that a path can be extended. Also, it allows path rerouting to efficiently utilize the network resources. Since rerouting is restricted to certain types of handoff calls (handoff counter $=\gamma$ or rerouting cell is hit), the processing load is much less than that in the incremental reestablishment scheme.

In LEO satellite networks, Out of seven previous rerouting algorithms the SHW(short hop win) algorithm is preferable than the other schemes especially when the satellite has no buffer which attains the best throughput performance .Also by using of multicast routing algorithm the multicast trees provide delay exceeding the minimum propagation delay by at most $5.5 \%$ on the average. Multicast trees are multiple times shorter than the sum of Uni-cast paths. They also outperform trees generated by Protocol Independent Multicast (PIM), MOSPF, and Core Based Tree (CBT) scheme.

The comparison between the Handover schemes in LEO satellite networks shows that the Handover -independent IP mobility management scheme is better in performance of the future IP application considering that the mobility management between the terrestrial mobile networks and LEO MSS's, due to the dynamic movement of both end user which need a continuous binding update of the geographical locations information.

Future work in WATM networks includes extended the stochastic model to analyze : (1) the mobile-to-mobile connection scenario;(2)the multicast connection in which a group of mobile users are communicating with each other; and (3)the path optimization problem with Quality of service constraints.

\section{REFERENCES}


[1] J.L, R.Yates and D.Raychaudhuri, "Performance Analysis of Path Rerouting Algorithms for Handoff Control in Mobile ATM Networks" IEEE Journal on selected areas in communications, VOL.18.NO.3. March 2000.

[2] F.Akyildiz, M.NAIRand Wang, "Mobility Management in Next-Generation Wireless Systems" PROCEEDING OF IEEE, VOL.87, NO 8, AUGUST 1999.

[3]G.Tham, J.Mai and L.Wong, "A QOS-Based Routing Algorithm for PNNI ATM Networks" National University of Singapore 2001.

[4]M.Marsanand A.Fumagali, "Performance Models of Handover Protocols and Buffering Policies in Mobile Wireless ATM Networks" IEEE Transaction on Vehicular Technology.VOL, 50.NO.4July 2001.

[5]K.Chan,S.Chan andE.Wong, “An Efficient Handoff Management scheme For Mobile Wireless ATM Networks " IEEE Transaction on Vehicular Technology ,VOL.49.NO.3.May 2000.

[ 6]V.Wong, M.Lewis and V.Leung, "Stochastic Control of Path Optimization for InterSwitch Handoff in Wireless ATM Networks" IEEE/ ACM Transaction on networking. VOL.9.NO.3 JUEN 2001.

[7]Y.Hu and V.Li, "Satellite -Based Internet: A Tutorial "IEEE Communication Magazine, March 2001.

[8]J.Bao and L.Tong, "Protocol-Aided Channel Equalization in Wireless ATM" IEEE Journal on selected areas in Communications.VOL.18.NO.3. March 2000.

[9] R, Ramjee and F.La Prta and D.Towsley, "Performance Evaluation of connection rerouting schemes for ATM based wireless networks" IEEE Transaction .on Networking, VOL.6.NO.3. June 1998.

[10]E.Ekici, F.Akyildiz and D.Bender, "Multicast Routing Algorithm for LEO Satellite IP Networks" (IEEE /ACM Transaction on networking, VOL.10.NO.2. Apr. 2002).

[11]F. Akyildiz and Seong-Ho Jeong, "Satellite ATM Networks: A Survey" IEEE, communication magazine, July 97.

[12]J.Sun and E.Modiano, "Routing Strategies for Maximizing Throughput in LEO Satellite Networks"IEEE Journal, on selected areas VOL.22.NO.2 Feb.2004.

[13]M.Werner, C.Delucchi and J.De Ridder, "ATM -based routing in LEO/MEO satellite ATM networks with intersatellite links" IEEE journal, on selected areas in Communication.VOL.15.NO.1 January 1997.

[14] A.Xhafa and O.K.Tonguz, "Dyanamic Priority Queuing of Handover calls in Wireless Networks: An Analytical Framework" IEEE Journal on selected area in Communication VOL 22 NO.5. JUNE 2004.

[15]M.Leo and M.Luglio, "Intersegment Handover between Terrestrial and Satellite Segment: Analysis and Performance Evaluation through Simulation"IEEE Transaction on Vehicular Technology VOL 50.No.3, MAY 2001.

[16]E.Del, R.Fantacci and G.Giambene, "Different Queuing Policies for Handover Requests in Low Earth Orbit Mobile Satellite Systems." IEEE Transaction on Vehicular Technology VOL 48.NO.2 March 1999.

[17]H.Tsunoda, K.Ohta and Y.Nemoto, "Supporting IP/LEO Satellite Networks by Handover- Independent IP Mobility Management" IEEE Journal on selected areas in communications February. 2000) . 